a wise choice of contributions for the enlightenment of those who wish to obtain as comprehensive a view as possible of the whole problem.

The particular solutions arrived at, which are quite properly advanced with caution, differ considerably ; but these are of less significance than the arguments that lead up to them. The essays should therefore be read in full if the greatest benefit is to be derived, but an indication of the conclusions may be of some interest. M. Scriven (Minnesota), from epistemological reasoning, concludes that "no verifiable claim can be made either that the Universe has a finite age or that it has not. We may still believe that there is a difference between these claims : but the difference is one that is not within the power of science to determine, nor will it ever be". J. T. Davies (London) provisionally accepts the age (about $4 \times 10^{9}$ years) given by linear extrapolation from the nebular recession hypothesis as being "the simplest interpretation of the observations and as being in excellent numerical accord with the age from independent investigations" of particular constituents of the universe. E. J. Öpik (Armagh) accepts the agreement (in general order of magnitude) of the ages given by various phenomena as suggesting an age not exceeding $6 \times 10^{9}$ years for the universe "in its present form and content". G. J. Whitrow (London) also lays stress on this agreement, which "strongly suggests that the universe has a finite age of some 4,000 million years". R. Schlegel (Michigan) prefers the view that the universe is "atemporal" - that is, the concept of time is inapplicable to it as a whole. He distinguishes this from the viow that the age of the universe is infinite, for this would be a particular solution for a universe to which the time concept is applicable. B. Abramenko (Mannheim), though admitting that conclusions must be based on observational evidence which at present is inadequate to settle the matter finally, favours the view that time, like space, is curved, so that physical processes occur in cycles. There is then no such thing as the age of the universe as a whole, and the indications of age from the various phenomena relate to the length of one cycle in the infinite succession of cycles. Unlike most of the writers, however, he attaches more significance to the differences between these indications than to their agreement.

All the more prominent systems of cosmologygeneral relativity, kinematical relativity, the 'steady state' theory involving continuous creation-are given some attention, but it is noteworthy that none of the essayists writes specifically from the point of view of any of them ; indeed, the objective character of the approach throughout is a praiseworthy feature of the collection. One point, however, would repay further elucidation. More than one writer is evidently under the impression that, in the 'steady state' theory, nebulæ are assumed continually to pass out of the region of possible observation, at a finite distance from the observer, through attaining and surpassing the velocity of light. Recent correspondence in The Observatory (74, 172; August 1954), however, suggests that this may be a misreading, notwithstanding that it is the apparent purport of the papers in which the theory was originally presented, and that in fact the theory assumes that the region observable in principle is infinite in extent, no nobula ever disappearing from it. It is very desirable that some clear statement on this point should be made by the authors of the theory, to prevent further misunderstanding.

\section{ROYAL OBSERVATORY, CAPE OF GOOD HOPE}

\section{REPORT FOR 1953}

$T$

HE report for 1953 of the Royal Observatory, Cape of Good Hope*, shows that an extensive programme of painting and repairs to the Observatory has been carried out, and dilapidations which accumulated during the war period have been practically overcome. Redecorations, externally and internally, have been effected in a number of buildings and at the time of writing the report only the now office buildings, a residence and a few other minor items required attention. In addition, electrical work has been carried out in some of the residences, a few extra trees have been planted, and it is hoped that a new hedge planted near the entrance gates will eventually provide some protection from the prevailing south-east wind.

The reversible transit circle has been in regular use and has required little special maintenance; observations were made with it on 155 days, 187 evenings and 113 mornings. The fitting of modern roller and thrust bearings to the declination axis of the Victoria telescope was completed in January, and the new cell made by Messrs. Cox, Hargreaves and Thomson for the 24-in. lens was then installed; this cell has resulted in a marked improvement in the performance of the lens. A number of alterations have been made in other ways, among which may be mentioned the replacement of the old windscreen by a new all-canvas screen which allows observations to be made within a few degrees of the horizon instead of $30^{\circ}$ as with the previous arrangement. The telescope was used on fifty-seven nights for photoelectric observations, sixty-one nights for direct photography, and the astrometric camera that is mounted on it on forty-two nights. The astrographic telescope, which was in continuous service, was used on one hundred and sixty-five nights for photoelectric photometry, on five nights for direct photography and on forty-eight nights for photography with the photometric cameras mounted on it. This telescope, while still behaving fairly well in spite of its age, requires a thorough overhaul in its drive and clamping mechanism, and, in addition, the axles and bearings of the wheels carrying the dome are badly worn and will shortly need renewing.

The 7-in. telescope and the tower telescope have been used for observing occultations, and the former has also been used for visitors. The tower telescope is not in good condition as the old stand is unsteady, and the lens requires repolishing. The 6 -in. telescope has been in regular use for observing long-period variable stars, and has also been used for visitors and for observing occultations. The photoheliograph has been used to take two photographs daily of the sun. The camera is in fair condition; but the stand is old, the polar axis unsteady and the clock drive unreliable. A few minor adjustments and alterations were necessary on the photometric cameras mounted on the astrographic telescope in November 1952, and the timing device was completed and brought into use. The astrometric (or wide-angle lens) camera which was dismounted from the astrographic telescope in 1952 was mounted on the Victorian teleseope

* Report of Her Majesty's Astronomer at the Cape of Good Hope to the Secretary of the Admiralty for the Year 1953. Pp. 11. (Cape of Good Hope: Royal Observatory. 1954.) 
when the work on the declination axis had been completed, and was used on forty-two nights during 1953.

Many other matters are dealt with under the following headings: observations and reductions; stellar photometry; Radcliffe section; meteorological observations; publication of results ; personnel establishment; and miscellaneous. Under the first of these is included an account of the parallax programme, and, although the observations for the main part of the parallax programme were completed in 1951, a few extra plates were needed to complete the determination of the proper motions in declination. Since the start of the programme in 1926, the parallaxes of 1,792 stars have been determined, while 3,508 plates are waiting to be measured for parallax and a few hundred for proper motion. Parallaxes were derived for thirty-two stars, none of which had a parallax previously derived at the Cape. Under the heading of stellar photometry is included work on bright stars, and reference is made in this to the new bright star programme which includes all stars in the Yale Bright Star Catalogue between dec. $-4^{\circ}$ and $-64^{\circ}$, together with a number of fainter stars from the radial velocity and parallax lists. Observations are being made in two colours with the photoelectric photometer attached to the astrographic refractor. The stars used in the previous programme, that is, those brighter than $H R 5 \cdot 0$, are used as standards, observations being made, as previously, at $30^{\circ}$ zenith distance. Under the heading of miscellaneous, Dr. R. H. Stoy, H.M. Astronomer at the Cape, refers to his two short trips overseas, the first to attend the conference on the co-ordination of galactic research, which was held at Groningen, and the second to attend two conferences in the United States, one being on photoelectric photometry, and the other on astrometry. "These three conferences," remarks Dr. Stoy, "relating as they did directly to the work of this Observatory, proved to be very helpful in suggesting the most useful programmes for future work."

\section{THE FUNCTION OF HYDROXYPROLINE IN COLLAGENS}

\section{By DR. K. H. GUSTAVSON}

Swedish Tanning Research Institute, Stockholm

\section{Introduction}

$\mathrm{T}$ HE degree of intermolocular and interfibrillar stability of collagen to hydrothermal influence, that is, its resistance to heat in the fully hydrated state, is most conveniently measured by the temperature at which instantaneous shrinkage of the collagen fibres takes place (thermal shrinkage). In earlier investigations, it was found that the shrinkage temperature, $T_{\varepsilon}$, of collagen from fish skin (Teleostei) is much lower than that of mammalian collagen, specimens of cod fish and of bovine skin mainly being investigated. Thus, native collagen of cod fish skin shrinks at temperatures of about $40^{\circ} \mathrm{C} .1,2$, when freely suspended, whereas bovine skin requires temperatures of about $65^{\circ} \mathrm{C}$. For collagen of the skin of eel and freshwater fishes from Swedish lakes, $T_{s}$ was found to be in the range $55-57^{\circ} \mathrm{C} .{ }^{2}$. Regarding the hydrothermal stability of the corium, the fishes form two distinct groups: (1) the coldand deep-water fishes for which $T_{\delta}$ of their skins is in the range $37-45^{\circ} \mathrm{C}$.; and (2) the warm- and surface-water fishes with values of $50-57^{\circ} \mathrm{C} .{ }^{3}$. During the past fifteen years, the shrinkage temperatures of a large number of skins from various species of salt- and fresh-water fishos in Swedish waters have been determined ${ }^{1-7}$. Independently, Takahashi and Tanaka ${ }^{8}$ have determined the shrinkage temperature of skin collagen from a great number of fishes in Japanese waters, and they also find the values to fall into two distinct temperature-ranges.

\section{Earlier Findings}

It was suggested in the early work on cod fish collagen $1,2,4$, at a time when data for the amino-acid composition of mammalian collagen were far from being quantitative and no reliable analysis of the amino-acid make-up of fish collagens was available, that the marked difference in hydrothermal stability. of bovine skin and cod-fish skin was probably not due to different chemical composition (amino-acids) but rather was a problem of different intermolecular stabilization of the two main classes of collagen. Thus, in the final analysis, it should be a question of molecular architecture and organization.

The following differences in chemical behaviour of the collagens of skins of mammals and teleosts (Gadus) have been found earlier-7,9-12. Collagen of fish skin is more easily gelatinized than that of mammalian skin. The former is also more extensively degraded and solubilized by acids and alkalis and by trypsin than native bovine $\operatorname{skin}^{1,2}$, which is practically unattacked by trypsin ${ }^{13,14}$. Thermal denaturation of fish collagen does not increase its reactivity towards large molecules, mainly interacting with proteins by means of co-ordinate valency forces (hydrogen bonding), for example, vegetable tannins, while the fixation of these agents by collagen of bovine origin is drastically increased by denaturation of the protein ${ }^{4-7}$. The reactivity of agents principally reacting with proteins by means of electrovalency is practically identical for both types of collagen, native as well as denaturated ${ }^{4-7}$.

Irreversibly fixed polyacids show distinctly different effects on the two types of collagen. The bovine type is not markedly changed, and is in some respects stabilized by these agents, whereas cod skin is practically destroyed by irreversible uptake of the polyacids, $T_{s}$ is much decreased and the fibre strength is completely lost ${ }^{9}$. Additional characterization has been obtained from investigations of the effect of anionic detergents such as sodium dodecyl sulphate in 5 per cent aqueous solution. Cod skin was largely solubilized, whereas no permanent alteration of bovine skin was found in these treatments. The unifunctional, non-swelling naphthalene$\beta$-sulphonic acid and polymetaphosphoric acids show the same differences in their action on the two forms of collagen ${ }^{10}$.

In view of these observations and the fact that the acid-and alkali-binding capacities of the two types of collagen were identical, it was suggested that the difference between the bovine and teleost (cod fish) collagens should be principally one of molecular organization, the low degree of stability of the fish-skin collagen being ascribed to a less developed 